\title{
Local consolidative therapy for oligometastatic patients with stage IV non-small cell lung cancer may improve survival, but unanswered questions remain
}

\author{
Jose M. Pacheco ${ }^{1}$, Drew Moghanaki ${ }^{2,3}$ \\ ${ }^{1}$ Division of Medical Oncology, Department of Internal Medicine, University of Colorado Anschutz Cancer Center, Aurora, CO, USA; ${ }^{2}$ Department \\ of Radiation Oncology, Emory University School of Medicine, Winship Cancer Institute, Atlanta, GA, USA; ${ }^{3}$ Department of Radiation Oncology, \\ Atlanta Veterans Affairs Health Care System, Decatur, GA, USA \\ Correspondence to: Jose M. Pacheco. University of Colorado Cancer Center, 1665 Aurora Court, Room 5309, Mail Stop F704, Aurora, CO 80045, \\ USA. Email: jose.m.pacheco@ucdenver.edu. \\ Comment on: Gomez DR, Tang C, Zhang J, et al. Local consolidative therapy vs. maintenance therapy or observation for patients with oligometastatic \\ non-small-cell lung cancer: long-term results of a multi-institutional, phase II, randomized study. J Clin Oncol 2019;37:1558-65.
}

Submitted Jun 13, 2019. Accepted for publication Jun 15, 2019.

doi: $10.21037 /$ tlcr.2019.06.07

View this article at: http://dx.doi.org/10.21037/tlcr.2019.06.07

\section{Introduction}

Oligometastatic disease refers to a limited number of metastatic sites that are either "synchronous" when presenting at the time of initial diagnosis or "metachronous" when arising following therapy of the primary tumor. It is hypothesized that the biology of oligometastatic tumors may be different than that of tumors with more widespread metastases (1-3). As such, treatment of oligometastatic sites with local consolidative therapy (LCT) is a topic of interest, and has been increasingly offered in clinical practice. While there is no single definition of this state, it is commonly defined as up to $3-5$ sites of metastases (excluding the primary lesion) following induction systemic therapy (1-6).

In patients receiving chemotherapy for non-small cell lung cancer (NSCLC), it has been observed that the most common sites of progression are within the original disease sites (1-3,6). Patients with oligometastatic disease remaining after induction systemic therapy are believed to harbor treatment resistant clones in these sites which may not be effectively managed with subsequent systemic agents (7). In these cases, there is a widow of opportunity to eradicate radiographically visible disease sites with LCT and delay further progressive disease, and perhaps even help patients live longer $(1,8,9)$.

Initially, highly select patients with limited metastases were treated off trial with LCT to all visible disease sites, including to the primary tumor (10). Later, retrospective observational studies and single arm prospective trials suggested a potential survival benefit with this approach (11-14). In a meta-analysis of 757 NSCLC patients with or without prior chemotherapy, and having 1-5 sites of metastases, the delivery of LCT to all visible disease sites (62\% surgery and $38 \%$ radiation) was associated with a 5 -year overall survival (OS) rate of $29.4 \%$ (15). When considering the historical 5 -year OS rate of $2 \%$ in stage IV NSCLC patients, this approach received widespread attention (16). However, this meta-analysis was subject to selection bias as it did not include randomized prospective data. Additionally, the historical rates included patients with any number of metastatic sites, and as such the patients treated with LCT for oligometastatic disease may have simply done better because their disease burden was lower. Subsequently, randomized clinical trials were initiated to more rigorously evaluate the role of LCT vs. standard systemic therapy/observation in patients with oligometastatic disease following induction chemotherapy (4-6).

\section{Gomez et al.'s trial}

This was a phase II randomized trial of LCT $v$ s. maintenance therapy/observation (MT/O) for patients with oligometastatic NSCLC who had either a partial response 
(PR) or stable disease (SD) after 3 months of initial systemic therapy. First line therapy consisted of one of the following regimens: 4 cycles of platinum-doublet chemotherapy, erlotinib or another approved frontline epidermal growth factor receptor (EGFR) inhibitor for patients with EGFR activating mutations or crizotinib for patients with anaplastic lymphoma kinase (ALK) fusions. To be eligible for enrollment, patients had to have $\leq 3$ metastatic sites, excluding the primary lesion, after completion of initial systemic therapy. Intrathoracic nodal involvement (mediastinal or supraclavicular) was counted as one site of disease, regardless of number of involved lymph nodes. LCT had to be administered to all visible disease sites and consisted of stereotactic ablative radiotherapy (SABR), hypofractionated radiotherapy, concurrent chemoradiation or surgery \pm radiation. Chemotherapy was held during LCT except in patients who received concurrent chemoradiation. Information was not available to determine if targeted therapy was continued concurrently with LCT in patients with EGFR and ALK alterations (5).

Enrollment to this trial was stopped early due to an improved progression free survival (PFS) with LCT that was reported in 2016 (4). A more recent update by Gomez et al., with a median of 38.8 months of follow-up time, demonstrated PFS of 14.2 months for LCT vs. 4.4 months for $\mathrm{MT} / \mathrm{O}, \mathrm{P}=0.022$. However, the highlight of this trial was a significantly improved OS for the group that received LCT, median OS 41.2 months (95\% CI, 18.9-not reached) vs. 17.0 months (95\% CI, 10.1-39.8) for MT/O, $\mathrm{P}=0.017$. It's notable that this OS benefit was seen despite many fewer patients receiving maintenance systemic therapy following LCT $(20 \%$ vs. $83 \%$ on the MT/O arm). Significantly higher rates of grade $\geq 3$ toxicity were not observed with LCT (5).

It was once believed that oligometastatic NSCLC did not benefit from local therapies and that systemic therapies should be preferentially utilized. However, the findings by Gomez et al. are contributing to a paradigm shift in the management of oligometastatic disease. This is in part due to the suggestion that LCT may alter the natural history of oligometastatic NSCLC, as reflected by a significant delay in the time to development of new metastases (median 14.2 months with LCT vs. 6.0 months with $\mathrm{MT} / \mathrm{O}, \mathrm{P}=0.11$ ). The data also revealed a prolongation of post-progression OS (median 37.6 months with LCT vs. 9.4 months with MT/O, $\mathrm{P}=0.034$ ), which was seen despite a numerically higher percentage of patients on the MT/O arm receiving post-progression LCT at $45 \%$ when compared to $32 \%$ in the LCT arm (5).
It deserves emphasis that the patients in this study were randomized after 3 months of first line systemic therapy. Baseline characteristics at randomization were similar between the groups, arguing against imbalances biasing outcomes. The number of metastatic lesions prior to starting systemic therapy for patients on each study arm was not provided, which leaves questions about whether there were significant differences between arms in this potential prognostic factor $(4,5)$. While metachronous metastases have been associated with better outcomes, only 3 of 49 patients on this study had metachronous metastases, so it is unlikely that imbalances in this prognostic factor affected the study conclusions $(3,5)$. Information is not available as to whether differences in molecular markers existed between the study arms which could have affected prognosis and/or availability of subsequent therapies. More patients on the LCT arm received subsequent systemic therapies post-progression (52\%) when compared to the MT/O arm (33\%), and whether this was reflective of a benefit from LCT or simply an imbalance between the groups is unclear $(4,5)$.

While these phase II data are exciting, there remain challenges when trying to incorporate the results into routine practice. The study was small with only 25 patients on the LCT arm and 24 patients on the MT/O arm. None of the patients on this trial received first line pembrolizumab or chemo-immunotherapy. There were also multiple LCT modalities utilized, and whether one is more or less effective remains unclear. Additionally, the trial was conducted at only three academic medical centers, and how effective this practice would be in the broader oncology community is unknown $(4,5)$.

\section{Current state of consolidation radiation for oligometastatic NSCLC}

The aforementioned trial by Gomez et al. was the first randomized prospective trial conducted exclusively in patients with oligometastatic NSCLC that demonstrated an OS benefit with LCT (5). Another randomized phase II trial focusing on this same patient population has evaluated LCT $v s$. maintenance systemic therapy following initial platinumdoublet chemotherapy, but has yet to report OS results. This latter trial was conducted at the University of Texas Southwestern (UTSW) medical center. The type of LCT was more consistent between patients on this trial when compared to the patients treated with LCT on the trial by Gomez et al. All patients on the LCT arm of the UTSW trial received SABR at similar biologically equivalent doses 
to metastatic sites and hypofractionated radiotherapy to the primary lesion for cases where SABR could not be safely delivered. No patients in this trial underwent concurrent chemoradiation or surgery. Patients had to have $\leq 5$ metastases (excluding the primary tumor) following 4-6 cycles of induction platinum-doublet chemotherapy, with the majority of patients having $\leq 3$ metastases at this point. Only patients with PR or SD to initial systemic therapy could enroll, and all sites of visible disease had to be treated with LCT on the experimental arm (6). Similarly to the Gomez study, trial enrollment was stopped early due a benefit with LCT (4-6). The UTSW trial suggested improved PFS with LCT, median PFS 9.7 months for LCT $(\mathrm{n}=14)$ vs. 3.5 months for maintenance therapy $(\mathrm{n}=15)$, HR 0.30 (95\% CI, 0.113-0.815) and $\mathrm{P}=0.01$. Toxicity was similar between the two treatment groups on the UTSW study (6).

It is notable that patients with EGFR and ALK molecular alterations were excluded from the UTSW trial, and there were only 8 patients with these targetable oncogenes on the trial by Gomez et al. (4-6). A retrospective study of LCT (surgery and/or radiation) for responding patients with $\leq 5$ metastases within 2 months of starting a first line EGFR inhibitor suggested an OS benefit for LCT in patients with EGFR activating mutations (17). However, the lack of randomized prospective data makes it difficult to determine the potential survival benefits of LCT in patients with EGFR activating mutations or patients with other oncogene drivers for which there are available targeted therapies. Thankfully, a pair of randomized trials are evaluating the benefit of LCT following initial treatment with tyrosine kinase inhibitors in patients with EGFR activating mutations (NCT03410043 and NCT03256981).

Based on the evidence above, the National Comprehensive Cancer Network (NCCN) recommends consideration of LCT to all sites of disease in patients with stage IV NSCLC who have PR or SD to frontline systemic therapy and up to 3-5 oligometastatic sites. This recommendation includes patients presenting with oligometastatic disease in the central nervous system (CNS) (18). It is recommended to treat any residual intrathoracic disease that is visible following initial systemic therapy with LCT, and not just the distant oligometastatic sites $(4-6,18)$.

\section{Future directions and remaining questions}

It is likely LCT in stage IV NSCLC will be increasingly used. However, there are many questions to be answered in order to optimize the utilization of this treatment modality. More specifically, as with any therapy we need to develop ways to select which patients are more likely to benefit. Below, we discuss some of these important questions and potential selection approaches.

It is unclear what the upper limit of oligometastatic sites is that can be effectively treated with LCT. Traditionally, NSCLC trials have defined oligometastatic disease as up to 3-5 sites of metastases following induction systemic therapy in patients with PR or SD to such treatment (4-6). However, ongoing trials [e.g., the SABR-COMET 10 trial (NCT 03721341)] are examining whether more than 5 oligometastatic sites may be effectively treated with LCT.

Next, could there be a role for circulating tumor cells (CTCs) and/or highly sensitive circulating tumor DNA (ctDNA) testing to help select patients with oligometastatic disease that are more or less likely to benefit from LCT following induction systemic treatment? The premise of LCT for oligometastatic disease is that the burden of residual, resistant cellular clones that are present in limited numbers of sites following induction systemic therapy may be reduced or even eradicated with LCT $(2,3)$. Higher levels of detectable CTCs and/or ctDNA may be suggestive of a more systemic disease process which LCT to visible oligometastatic sites cannot successfully eradicate. On the other hand, CTCs and/or ctDNA testing could be useful in identifying treatment resistant clones that may benefit from LCT due to limited systemic options. Incorporation of these investigative methods into trials of LCT for oligometastatic disease could help advance the field by identifying populations more or less likely to benefit from LCT.

In addition to the promise of liquid biopsies, preclinical experiments and limited translational work in humans suggest the type of micro RNAs detected in metastatic sites could help determine whether patients will have a more aggressive metastatic spread or a less aggressive metastatic spread (1-3). There is also the possibility that more in depth DNA/RNA analysis of the primary tumor, metastatic sites and/or their associated microenvironment may help predict which NSCLC patients with oligometastatic disease are more likely to have an indolent course and hence potentially benefit more from LCT.

A separate question that remains unanswered is whether or not concurrent chemotherapy with conventional radiotherapy may offer similar benefits when compared to ablative doses of radiotherapy. In the UTSW trial, LCT only consisted of SABR, and systemic therapy was not administered concurrently (6). In contrast, in the Gomez et 
al.'s trial, some patients received concurrent chemoradiation for LCT $(4,5)$. Further research needs to be conducted to determine whether it is safe and potentially more effective to continue systemic therapies during radiation administered as LCT, as opposed to SABR, and if such a concurrent approach can be utilized for all approved systemic therapies (immunotherapy \pm chemotherapy or tyrosine kinase inhibitors).

Then, there is the question about whether LCT to oligometastatic sites will be similarly effective following initial immunotherapy-based regimens, as compared to the results seen following induction chemotherapy with platinum-based doublets. Some investigators believe that the benefit of LCT may be greater following these newer treatment modalities; however, reliable clinical data is lacking in this regard. In studies on colorectal cancer patients with limited liver metastases, it has been suggested that overexpression of genes involved in innate and adaptive immunity are associated with metastases that are more likely to be limited in number and virulence (19). Additionally, in preclinical models it has been suggested that the optimal cytotoxic effect of radiotherapy is dependent on the presence of active cytotoxic T-cells (20). Murine models suggest that the anti-tumor effects of ionizing radiation plus PD-1 axis inhibition are more effective than either modality by itself (21). A phase I trial evaluating 79 patients treated with anti-PD-1 therapy combined with radiation to $2-4$ sites demonstrated that $>90 \%$ of patients did not have progressive disease in lesions that received combined modality therapy, while less than $15 \%$ of patients had a response in non-irradiated sites (22).

Lastly, highly CNS penetrant tyrosine kinase inhibitors are now available for patients with certain molecular alterations. For these patients, whether LCT to oligometastatic CNS lesions is necessary following initial induction therapy is an important question. It is possible that for such patients' local therapy could be reserved for CNS salvage.

\section{Conclusions}

LCT appears to benefit some patients with oligometastatic stage IV NSCLC following initial systemic therapy (4-6). When considering LCT for this population, it is important to avoid considering clinical or molecular factors in isolation, as combinations of these may optimally identify patients most likely to benefit. Besides the presence of metachronous disease, other important clinical factors to consider include: time to development of metachronous disease, number of involved organs, evidence/or lack thereof for lymph node involvement and total tumor burden (1-3). Lastly it will be essential to try to distinguish which of the potential markers discussed above are simply prognostic of improved survival for patients with oligometastatic disease and which are truly predictive of improved outcomes with LCT. It is unlikely that the optimal integration of LCT into routine clinical practice will be as easy as simply administering it to all responding patients with up to 3-5 oligometastatic sites following initial systemic treatment. Improved selection criteria for LCT in stage IV NSCLC are needed.

\section{Acknowledgments}

None.

\section{Footnote}

Conflicts of Interest: JM Pacheco: Advisory board/consulting for AstraZeneca and Novartis. Honorarium from Genentech and Takeda. Research funding from Pfizer. D Moghanaki: Honorarium from Varian Medical Systems.

Ethical Statement: The authors are accountable for all aspects of the work in ensuring that questions related to the accuracy or integrity of any part of the work are appropriately investigated and resolved.

\section{References}

1. Weichselbaum RR. The 46th David A. Karnofsky Memorial Award Lecture: Oligometastasis-From Conception to Treatment. J Clin Oncol 2018. [Epub ahead of print].

2. Foster CC, Pitroda SP, Weichselbaum RR. Staging the Metastatic Spectrum Through Integration of Clinical and Molecular Features. J Clin Oncol 2019;37:1270-6.

3. Pitroda SP, Weichselbaum RR. Integrated molecular and clinical staging defines the spectrum of metastatic cancer. Nat Rev Clin Oncol 2019;16:581-8.

4. Gomez DR, Blumenschein GR Jr, Lee JJ, et al. Local consolidative therapy vs. maintenance therapy or observation for patients with oligometastatic non-smallcell lung cancer without progression after first-line systemic therapy: a multicentre, randomised, controlled, phase 2 study. Lancet Oncol 2016;17:1672-82. 
5. Gomez DR, Tang C, Zhang J, et al. Local consolidative therapy vs. maintenance therapy or observation for patients with oligometastatic non-small-cell lung cancer: long-term results of a multi-institutional, phase II, randomized study. J Clin Oncol 2019;37:1558-65.

6. Iyengar P, Wardak Z, Gerber DE, et al. Consolidative Radiotherapy for Limited Metastatic Non-Small-Cell Lung Cancer: A Phase 2 Randomized Clinical Trial. JAMA Oncol 2018;4:e173501.

7. Correa RJ, Salama JK, Milano MT, et al. Stereotactic Body Radiotherapy for Oligometastasis: Opportunities for Biology to Guide Clinical Management. Cancer J 2016;22:247-56.

8. Hellman S, Weichselbaum RR. Oligometastases. J Clin Oncol 1995;13:8-10.

9. Niibe Y, Hayakawa K. Oligometastases and oligorecurrence: the new era of cancer therapy. Jpn J Clin Oncol 2010;40:107-11.

10. Barney JD, Churhill EJ. Adenocarcinoma of the kidney with metastasis to the lung cured by nephrectomy and lobectomy. J Urol 1939;42:269-76.

11. De Ruysscher D, Wanders R, van Baardwijk A, et al. Radical treatment of non-small-cell lung cancer patients with synchronous oligometastases: long-term results of a prospective phase II trial (Nct01282450). J Thorac Oncol 2012;7:1547-55.

12. Hasselle MD, Haraf DJ, Rusthoven KE, et al. Hypofractionated image-guided radiation therapy for patients with limited volume metastatic non-small cell lung cancer. J Thorac Oncol 2012;7:376-81.

13. Milano MT, Katz AW, Zhang H, et al. Oligometastases treated with stereotactic body radiotherapy: long-term follow-up of prospectivestudy. Int J Radiat Oncol Biol Phys 2012;83:878-86.

14. Rusthoven KE, Kavanagh BD, Burri SH, et al. Multiinstitutional phase I/II trial of stereotactic body radiation therapy for lung metastases. J Clin Oncol 2009 Apr 1;27:1579-84.

15. Ashworth AB, Senan S, Palma DA, et al. An individual patient data metaanalysis of outcomes and prognostic factors after treatment of oligometastatic non-small-cell lung cancer. Clin Lung Cancer 2014;15:346-55.

16. Cetin K, Ettinger DS, Hei YJ, et al. Survival by histologic subtype in stage IV nonsmall cell lung cancer based on data from the Surveillance, Epidemiology and End Results Program. Clin Epidemiol 2011;3:139-48.

17. Xu Q, Zhou F, Liu H, et al. Consolidative Local Ablative Therapy Improves the Survival of Patients with Synchronous Oligometastatic NSCLC Harboring EGFR Activating Mutation Treated With First-Line EGFRTKIs. J Thorac Oncol 2018;13:1383-92.

18. Non-Small Cell Lung Cancer. Version 4.2019. Referenced with permission from the NCCN Clinical Practice Guidelines in Oncology (NCCN Guidelines ${ }^{\circledR}$ ) for Non-Small Cell Lung Cancer V4.2019. National Comprehensive Cancer Network, Inc., 2019. Available online: https://www.nccn.org/

19. Pitroda SP, Khodarev NN, Huang L, et al. Integrated molecular subtyping defines a curable oligometastatic state in colorectal liver metastasis. Nat Commun 2018;9:1793.

20. Lee Y, Auh SL, Wang Y, et al. Therapeutic effects of ablative radiation on local tumor require CD8+ $\mathrm{T}$ cells: changing strategies for cancer treatment. Blood 2009;114:589-95.

21. Deng L, Liang H, Burnette B, et al. Irradiation and antiPD-L1 treatment synergistically promote antitumor immunity in mice. J Clin Invest 2014;124:687-95.

22. Luke JJ, Lemons JM, Karrison TG, et al. Safety and Clinical Activity of Pembrolizumab and Multisite Stereotactic Body Radiotherapy in Patients With Advanced Solid Tumors. J Clin Oncol 2018;36:1611-8.
Cite this article as: Pacheco JM, Moghanaki D. Local consolidative therapy for oligometastatic patients with stage IV non-small cell lung cancer may improve survival, but unanswered questions remain. Transl Lung Cancer Res 2019;8(Suppl 4):S407-S411. doi: 10.21037/tlcr.2019.06.07 